\title{
Perfluoroalkyl substances exposure and thyroid hormones in humans: epidemiological observations and implications
}

\author{
Jung Eun Lee, BS, \\ Kyungho Choi, DVM, MPH, \\ $\mathrm{PhD}$
}

School of Public Health, Seoul National University, Seoul, Korea
Received: 20 March, 2017

Revised: 27 March, 2017

Accepted: 28 March, 2017

Address for correspondence:

Kyungho Choi, DVM, MPH, PhD

Department of Environmental

Health Sciences, School of Public

Health, Seoul National University,

1 Gwanak-ro, Gwanak-gu, Seoul 08826, Korea

Tel: +82-2-880-2738

Fax: +82-2-762-9104

E-mail: kyungho@snu.ac.kr

http://orcid.org/0000-0001-7460-

$792 X$
Thyroid hormones play crucial roles in normal neurodevelopment of fetus and child. Many chemicals can affect control and homeostasis of thyroid hormones, and eventually lead to various adverse health effects including neurodevelopmental disorders. Perfluoroalkyl substances (PFASs) are among the thyroid disrupting chemicals that can be encountered among general human population. Due to their unique physicochemical characteristics, PFASs have been used as surfactants and surface coating materials in many applications. Therefore, PFASs have been frequently detected in humans and environment worldwide. In cross-sectional studies using nationally representative general human populations of United States, several PFASs have shown significant associations with thyroid hormones. Moreover, among pregnant women and their infants, not only major PFASs such as perfluorooctane sulfonic acid and perfluorooctanoic acid, but also those with shorter or longer carbon chains showed significant associations with thyroid hormones. Often demographic characteristics such as sex, age, and disease status appear to influence the associations between PFASs exposure and thyroid hormones. In general, major PFASs showed hypothyroidism effects among pregnant women and infants. As 8 carbon based PFASs have been phased out, those with shorter or longer carbon chains have been used in growing amount as replacement. However, only limited information is available for their occurrences and toxicity among humans. Further investigations on these substituting PFASs are required. In addition, efforts are warranted to identify sources of and mitigate exposure to these thyroid disrupting chemicals especially during pregnancy and early stages of life.

Keywords: Perfluoroalkyl substances, Biomonitoring, Thyroid, Disruption, population, Pregnant women

\section{Introduction}

Neurodevelopmental disorders among childhood have increased significantly over the past several decades worldwide. In 2014, Centers for Disease Control and Prevention of United States estimated that one out of 68 children show autism spectrum disorder (ASD) ${ }^{1}$. This number is very high, compared to the old statistics, e.g., 1 out of 150 children in 2007, and 1 out of 2,500 in $1985^{2)}$. In addition, up to $20 \%$ of children, depending on country, exhibit attention deficiency hyperactivity disorders ${ }^{3}$. Aggressive diagnosis and reporting may explain this phenomenal upsurge of the neurodevelopmental disorders to certain extent. However, importance of environmental causes gains more recognition as determinants of these diseases among the scientists. For example, environmental contaminants are suggested as major causes of ASD, along with diagnostic accretion, greater awareness, and age of parents ${ }^{2}$. 
Thyroid hormones are involved in regulation of various processes over early brain development ${ }^{4}$. These hormones are involved in dendritic and axonal growth, neurogenesis, and myelination processes in developing fetus during pregnancy. After birth as well, these hormones are important in myelination of neurons. Therefore, even subclinical changes of thyroid hormones during these susceptible periods of life may lead to delays in neuropsychological development of children ${ }^{5}$.

Numerous observational studies support the importance of thyroid hormones in neurodevelopment of children. Infants with low thyroxine (T4) showed modestly increase risk of ASD in large scale population study in California United States ${ }^{6}$. According to the observations of a prospective cohort study on Dutch mother-child pairs $(\mathrm{n}=3,839)$, when the levels free triiodothyronine (T3) of mother were too high or low, the average intelligence quotient (IQ) of her child was lower by $1.4-3 \cdot 8^{7}$. As fetal thyroid gland is not sufficiently developed until second trimester of pregnancy, development of fetus will be likely influenced by maternal thyroid hormones. Therefore, the factors that will affect not only thyroid hormones of the infants but also those of pregnant mothers can affect the IQ and neurodevelopment of the children.

Thyroid disrupting chemicals (TDCs) are suggested to be responsible for, to certain extent, rapid increase of neurodevelopmental diseases worldwide. Numerous chemicals are reported to disrupt thyroid hormone balances. These chemicals include phthalates and phenolic compounds that are widely used in daily life, and persistent organic compounds such as organochlorine pesticides (OCPs), polychlorinated biphenyls (PCBs), polybrominated diphenylethers (PBDEs), and perfluoroalkyl substances (PFASs) ${ }^{8)}$.

\section{Perfluoroalkyl substances}

PFASs are reported to disrupt thyroid hormone balances by increasing metabolic excretion and inhibiting synthesis of the hormones ${ }^{9}$. Unlike other persistent organic compounds which preferentially partition into lipid, PFASs are unique as these compounds can resist both water and oil. This characteristic is due to their structure with perfluorinated carbon tail and

Table 1. Perfluoroalkyl substances - classification and physicochemical characteristics

\begin{tabular}{|c|c|c|c|c|c|}
\hline \multirow{2}{*}{ Compound } & \multirow{2}{*}{ CAS RN } & \multirow{2}{*}{$\begin{array}{c}\text { Molecular weight } \\
(\mathrm{g} / \mathrm{mol})\end{array}$} & \multirow{2}{*}{$\log K_{\text {ow }}{ }^{\mathrm{b})}$} & \multicolumn{2}{|c|}{ Serum elimination half-lives } \\
\hline & & & & Male & Female \\
\hline \multicolumn{6}{|l|}{ Perfluoroalkyl carboxylic acids (PFCAs) } \\
\hline \multicolumn{6}{|c|}{ Short chain PFCAs $\left(\mathrm{CnF}_{2 n+1} \mathrm{COOH}, \mathrm{n}<7, \mathrm{PFCAs}\right)^{a)}$} \\
\hline Perfluorobutanoic acid (PFBA) & $375-22-4$ & 214.04 & $3.391 \pm 0.597$ & $\begin{array}{l}{ }^{*} 68 \pm 35 \mathrm{hr}^{\mathrm{c})} \\
{ }^{+} 6 \mathrm{hr}(\mathrm{IV})^{c}, 9 \mathrm{hr}(\text { Oral })^{c)}\end{array}$ & $\begin{array}{l}{ }^{*} 87 \pm 31 \mathrm{hr}^{\mathrm{c})} \\
{ }^{\dagger} 1 \mathrm{hr}(\mathrm{IV})^{c}, 2 \mathrm{hr}(\text { Oral })^{c)}\end{array}$ \\
\hline Perfluoropentanoic acid (PFPeA) & $2706-90-3$ & 264.05 & $4.391 \pm 0.657$ & - & - \\
\hline Perfluorohexanoic acid (PFHxA) & $307-24-4$ & 314.05 & $4.985 \pm 0.710$ & ${ }^{*}<28$ days ${ }^{\mathrm{d})},{ }^{\dagger} 1 \mathrm{hr}(\mathrm{IV})^{\mathrm{g})}$ & ${ }^{\dagger} 0.4 \mathrm{hr}(\mathrm{IV})^{\mathrm{g})}$ \\
\hline Perfluoroheptanoic acid (PFHpA) & $375-85-9$ & 364.06 & $5.714 \pm 0.759$ & ${ }^{\dagger} 0.10$ days $(\mathrm{IV})^{\mathrm{h})}$ & ${ }^{\dagger} 0.05$ days $(\mathrm{IV})^{\mathrm{h})}$ \\
\hline \multicolumn{6}{|c|}{ Long chain PFCAs $\left(\mathrm{CnF}_{2 n+1} \mathrm{COOH}, \mathrm{n} \geq 7, \mathrm{PFCAs}\right)$} \\
\hline Perfluorooctanoic acid (PFOA) & $335-67-1$ & 414.07 & $6.444 \pm 0.806$ & $\begin{array}{l}{ }^{*} 3.8 \pm 1.7 \mathrm{yr}^{\mathrm{e})} \\
{ }^{+} 5.63 \text { days }(\mathrm{IV})^{\mathrm{h})}\end{array}$ & $\begin{array}{l}\left.{ }^{*} 3.3 \mathrm{yr}^{\mathrm{e}}\right) \\
{ }^{\dagger} 0.08 \text { days }(\mathrm{IV})^{\mathrm{h})}\end{array}$ \\
\hline Perfluorononanoic acid (PFNA) & $375-95-1$ & 464.08 & $7.174 \pm 0.844$ & $\begin{array}{l}{ }^{\dagger} 29.5 \text { days }(\text { IV })^{\mathrm{h})}, 30.6 \\
\text { days }(\text { Orall) }\end{array}$ & $\begin{array}{l}{ }^{\dagger} 2.44 \text { days }(\mathrm{IV})^{\mathrm{h})}, 1.4 \text { days } \\
(\text { Oral })^{\mathrm{i}}\end{array}$ \\
\hline Perfluorodecanoic acid (PFDA) & $335-76-2$ & 514.08 & $7.904 \pm 0.860$ & ${ }^{\dagger} 39.9$ days $(\mathrm{IV})^{\mathrm{h})}$ & ${ }^{\dagger} 58.6$ days $(\mathrm{IV})^{\mathrm{h})}$ \\
\hline Perfluorododecanoic acid (PFDoDA) & $307-55-1$ & 614.1 & $9.363 \pm 0.888$ & - & - \\
\hline Perfluorotridecanoic acid (PFTrDA) & 72629-94-8 & 664.11 & $10.093 \pm 0.901$ & - & - \\
\hline Perfluorotetradecanoic acid (PFTeDA) & $376-06-7$ & 714.11 & $10.823 \pm 0.914$ & - & - \\
\hline \multicolumn{6}{|l|}{ Perfluoroalkyl sulfonic acids (PFSAs) } \\
\hline \multicolumn{6}{|l|}{ Short chain PFSAs $\left(\mathrm{CnF}_{2 n+1} \mathrm{SO}_{3} \mathrm{H}, \mathrm{n}<6, \mathrm{PFSAs}\right)$} \\
\hline Perfluorobutane sulfonic acid (PFBS) & $375-73-5$ & 300.1 & $1.689 \pm 0.719$ & $\begin{array}{l}{ }^{*} 24 \pm 7 \text { days }{ }^{f)} \\
{ }^{\dagger} 5 \mathrm{hr}(\mathrm{IV})^{\mathrm{f}}, 5 \mathrm{hr}(\text { Oral })^{\mathrm{f})}\end{array}$ & $\begin{array}{l}{ }^{*} 46 \text { days }^{\mathrm{f})} \\
{ }^{+} 4 \mathrm{hr}(\mathrm{IV})^{\mathrm{f}}, 8 \mathrm{hr}(\text { Oral })^{\mathrm{f}}\end{array}$ \\
\hline \multicolumn{6}{|l|}{ Long chain PFSAs $\left(\mathrm{CnF}_{2 n+1} \mathrm{SO}_{3} \mathrm{H}, \mathrm{n} \geq 6, \mathrm{PFSAs}\right)$} \\
\hline Perfluorohexane sulfonic acid (PFHxS) & $355-46-4$ & 400.11 & $3.053 \pm 0.814$ & $\begin{array}{l}{ }^{*} 8.2 \pm 5.1 \mathrm{yr}^{\mathrm{e})} \\
{ }^{+} 29 \text { days (IV) }\end{array}$ & $\begin{array}{l}{ }^{*} 12.8 \pm 0.6 \mathrm{yr}^{\mathrm{e})} \\
{ }^{\dagger} 2 \text { days (IV) }\end{array}$ \\
\hline Perfluorooctane sulfonic acid (PFOS) & $1763-23-1$ & 500.13 & $4.512 \pm 0.862$ & $\begin{array}{l}* 5.4 \pm 3.7 \mathrm{yr}^{\mathrm{e})} \\
{ }^{\dagger} 38 \text { days }(\text { Oral })^{\mathrm{k})}, 41 \text { day } \\
\left(\text { Oral) }{ }^{\mathrm{k})}\right.\end{array}$ & $\begin{array}{l}* 5.9 \pm 1 \mathrm{yr}^{\mathrm{e})} \\
\left.{ }^{\dagger} 62 \text { days (Oral) }\right)^{\mathrm{k})}, 71 \text { days } \\
(\text { Oral })^{\mathrm{k})}\end{array}$ \\
\hline Perfluorodecane sulfonic acid (PFDS) & $335-77-3$ & 600.14 & $5.972 \pm 0.891$ & - & - \\
\hline
\end{tabular}

CAS RN, chemical abstracts service registry number.

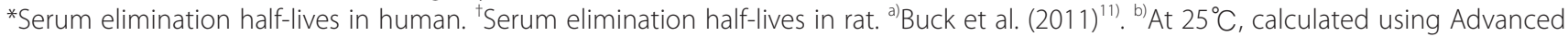

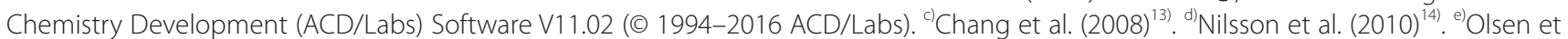

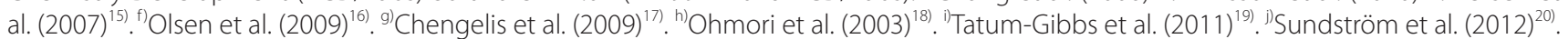
${ }^{k}$ Chang et al. $(2012)^{211}$. 
polar head ${ }^{10)}$. For this property, PFASs have been widely used as surfactants and surface coatings in various applications in industry and commerce since 1950s, such as textiles, food contact paper, cookware, carpets, cosmetics, photographic emulsifiers, lubricants, paints, fire-fighting foams, and food packaging ${ }^{11,12)}$.

PFASs are highly fluorinated synthetic aliphatic substances of which $\mathrm{H}$ substituents are replaced by $\mathrm{F}\left(\mathrm{CnF}_{2 \mathrm{n}+1}\right)$. Major PFASs are shown in Table $1^{11,13-21)}$ and Fig. 1 . By the component, PFASs are grouped into perfluoroalkyl sulfonic acids (PFSAs) and perfluoroalkyl carboxylic acids (PFCAs). Generally longer carbon chain PFASs, e.g., long-chain PFSAs (number of C $\geq 6$ ) and PFCAs (number of $C \geq 7$ ), are reported to be more bioaccumulative and persistent compared to the short-chain PFASs ${ }^{11)}$. Definition of long-chain is different for PFCAs and PFSAs, as PFSA with 6 carbons (perfluorohexane sulfonic acid $[\mathrm{PFHxS}])$ is more bioaccumulative than perfluorohexanoic acid (PFHxA).

Recently, heavily used long-chain PFASs such as perfluorooctane sulfonic acid (PFOS) have been phased out, and shorter carbon chain PFASs have been used as replacements in increasing amount ${ }^{22)}$. Therefore, the occurrence of the substitutes in both environment and biota has increased worldwide. In breastmilk samples of Korean lactating women $(\mathrm{n}=265)$, while perfluorooctanoic acid (PFOA) and PFOS were detected in $98.5 \%$ of the samples, short carbon chain PFASs such as perfluoropentanoic acid (PFPeA), PFHxA, and perfluoroheptanoic acid, were detected in high frequency ranging between $67.4 \%$ and $81.8 \%$. The levels of PFPeA and PFHxA in breastmilk were the greatest compared to the previous reports suggesting that these compounds that replace the long chain PFASs are used in increasing amount over time ${ }^{23)}$. Longer carbon chain PFASs (number of $\mathrm{C} \geq 9$ ) are used also in growing amount. Reflecting this trend, in Australia, the serum levels of PFOS and PFOA showed decreasing trend between 2002 and 2013, but perfluorododecanoic acid (PFDoDA) showed an increase ${ }^{24)}$.

\section{Biological half-lives of PFASs}

Because of the different composition and chain length, biological half-lives of PFASs vary by structure. Long-chain compounds like PFHxS, PFOS, and PFOA have longer half-lives than the PFASs with shorter carbon chains ${ }^{16)}$. For example, halflife of serum PFOA is estimated up to 8.5 years, but those for perfluorobutanoic acid and PFHxA are ranging between 3 and 28 days $^{13,14)}$. Biological half-lives of other short-chain PFASs are also expected to be relatively short.

\section{PFASs exposure and thyroid hormone disruption in general populations}

Association studies on general population without occupational exposure are accumulating. Several big scale studies employing nationally representative populations are also conducted. In addition, studies on susceptible human populations such as pregnant women and newborns or children are conducted. Reports on nationally representative human populations are mostly based on US National Health and Nutrition Examination Survey (NHANES). Among general US population, PFOS, PFOA, PFHxS, and perfluorononanoic acid (PFNA) are most frequently measured and reported. The exposure to these chemicals showed significant association with thyroid hormone levels (Table 2) ) $^{2529}$.

Among adults with current thyroid diseases, PFOA or PFOS were detected at relatively higher levels ${ }^{25}$. While many PFASs

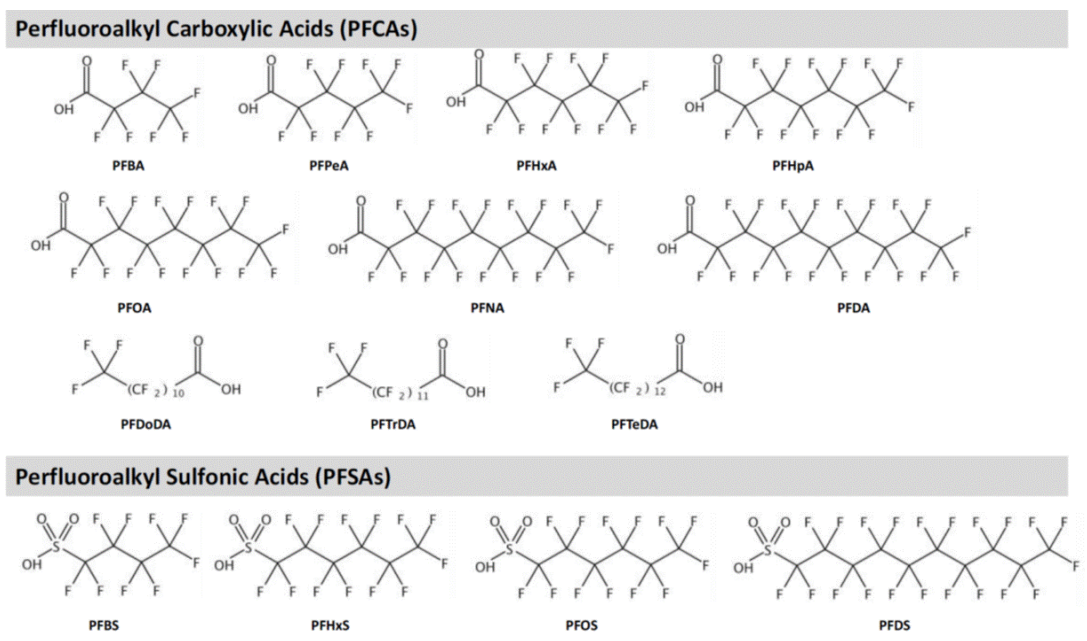

Fig. 1. Structures of major perfluoralkyl substances. PFBA, perfluorobutanoic acid; PFPeA, perfluoropentanoic acid; PFHXS, perfluorohexane sulfonic acid; PFHpA, perfluoroheptanoic acid; PFOA, perfluorooctanoic acid; PFNA, perfluorononanoic acid; PFDA, perfluorodecanoic acid; PFDoDA, perfluorododecanoic acid; PFTrDA, perfluorotridecanoic acid; PFTeDA, perfluorotetradecanoic acid; PFBS, perfluorobutane sulfonic acid; PFOS, perfluorooctane sulfonic acid; PFDS, perfluorodecane sulfonic acid. 
showed significant associations with thyroid hormone levels, the directions of association were different by $\operatorname{sex}^{26)}$, and age $\mathrm{e}^{27)}$. According to the survey 2007-2010, the PFOA and PFHxS levels showed positive associations with total thyroid hormones among female $(\mathrm{n}=509)$, however, among male population $(n=672)$, negative association was observed between PFHxS and free T4 (fT4) levels ${ }^{26)}$. Among the adolescents in the 2011-2012 survey, males $(n=158)$ showed positive associations between PFOS or PFNA and thyroid stimulating hormone (TSH), while females $(n=145)$ showed a negative association between PFOA and $\mathrm{TSH}^{27)}$. In the same survey round (NHANES, 2011-2012), effects of age were also observed. Female subjects of 20 to 40 years of age $(n=268)$ showed positive associations between study PFASs, i.e., PFOA, PFHxS, PFNA, or PFOS, and TSH. However, among females of 60 to 80 years of age, a negative association between PFHxS and TSH was observed ${ }^{27)}$. By levels of thyroid peroxidase (TPO) antibody (Ab) and urinary iodine, the association between PFASs and thyroid hormones differs ${ }^{28)}$. In NHANES 2007-2008, subjects with normal levels of TPOAb and urinary iodine $(n=1,012)$ exhibited a positive association between PFOA and free T3. However, among those who showed abnormal range of these markers $(n=26)$ negative associations were observed between PFHxS or PFOA and $\mathrm{fT} 4{ }^{28)}$. While it was not nationally representative sampling, among general
Korean populations ( $>12$ years of age, $\mathrm{n}=644$ ), Ji et al. ${ }^{30)}$ reported a negative association of perfluorotridecanoic acid with tT4, and a positive association with TSH, in a small industrial city of Siheung, Korea.

The influences of sex and age on the reported associations may be due to the biological half-lives of PFASs. The biological half-lives of major PFASs tend to be shorter among females, probably because of menstruation ${ }^{31)}$. The fact that post-menopausal women, e.g., women of 60-80 years of age, showed different direction of association compared to menstruating women $^{27)}$, may also support this hypothesis. In addition, the interaction between sex hormones and thyroid hormones cannot be excluded. Several human thyroid disorders are accompanied by changes in sex steroids ${ }^{32}$. In other animals, e.g., rainbow trout, estradiol exposure decreased activities of T4-outer ring deiodination (ORD) and rT3-ORD in liver, but increased activity of T3-inner ring deiodination in kidney in both sexes ${ }^{33)}$. In addition, estradiol treatment in immature rainbow trout, caused decrease of T3 in plasma ${ }^{34)}$.

As shown above, among general populations with various demographic characteristics, such as sex, age, and disease status, it is not easy to observe consistent directions of association. Moreover, potential effects of other TDCs that may present in the study populations cannot be ignored. Since the exposure

Table 2. Associations between exposure to PFASs and thyroid hormones among nationally representative populations of United States

\begin{tabular}{|c|c|c|c|c|c|}
\hline PFASS & Population age $(n)$ & Study period & Thyroid measure & Association & Reference \\
\hline $\begin{array}{l}\text { PFOA } \\
\text { PFOS }\end{array}$ & General population $(3,974)$ & 1999-2006 & Current thyroid disease & $\begin{array}{l}\text {-PFOA highest quartile shows odd ratio (OR) for } \\
\text { current thyroid disease of } 2.24 \text { ( } 95 \% \mathrm{Cl}, 1.38-3.65) \\
\text { in females, and } 2.12(0.93-4.82) \text { for male } \\
\text {-PFOS highest quartile shows OR for current thyroid } \\
\text { disease of } 2.68(1.03-6.98) \text { in males }\end{array}$ & Melzer et al. $(2010)^{25)}$ \\
\hline $\begin{array}{l}\text { PFOS, } \\
\text { PFOA, } \\
\text { PFDeA, } \\
\text { PFHxS, } \\
\text { PFNA }\end{array}$ & $>12$ years $(1,832)$ & $2007-2008$ & fT3, fT4, tT3, tT4, TSH & $\begin{array}{l}\text {-PFOA in positive associations with TSH and tT3 } \\
\text {-PFHXS in positive association with tT4 }\end{array}$ & Jain $(2013)^{29)}$ \\
\hline $\begin{array}{l}\text { PFOA, } \\
\text { PFHxS, } \\
\text { PFNA, } \\
\text { PFOS }\end{array}$ & $>18$ years $(1,525)$ & $2007-2008$ & fT3, fT4, tT3, tT4, TSH & $\begin{array}{l}\text {-Among population with normal range of TPOAb } \\
\text { and urinary iodine }(n=1,012), \text { PFOA in positive } \\
\text { association with fT3 } \\
\text {-Among population with abnormal range of TPOAb } \\
\text { and urinary iodine ( } n=26), \text { PFHxS and PFOA } \\
\text { in negative associations with fT4. All } 4 \text { PFSAs } \\
\text { in positive associations with } \mathrm{fT} 3 \text {, } \mathrm{tT} 3, \mathrm{fT} 3 / \mathrm{fT} 4 \text {, or TSH }\end{array}$ & Webster et al. (2016) \\
\hline $\begin{array}{l}\text { PFOA, } \\
\text { PFHxS, } \\
\text { PFNA, } \\
\text { PFOS }\end{array}$ & $\geq 20$ years $(1,181)$ & $2007-2010$ & fT3, fT4, tT3, tT4, TSH & $\begin{array}{l}\text {-Among female }(n=509), \text { PFOA in positive } \\
\text { association with tT3; PFHxS in positive associations } \\
\text { with tT4 and tT3 } \\
\text {-Among male ( } n=672) \text {, PFHxS in negative } \\
\text { association with fT4 }\end{array}$ & Wen et al. $(2013)^{26)}$ \\
\hline $\begin{array}{l}\text { PFOA, } \\
\text { PFHxS, } \\
\text { PFNA, } \\
\text { PFOS }\end{array}$ & $\geq 12$ years $(1,682)$ & $2011-2012$ & fT3, fT4, tT3, tT4, TSH & $\begin{array}{l}\text {-No associations observed in whole population } \\
\text {-Among females of } 20-40 \text { years of age }(n=268) \text {, } \\
\text { all PFSAs in positive associations with TSH. But in } \\
\text { females of } 60-80 \text { years of age, PFHxS in negative } \\
\text { association with TSH } \\
\text {-Among female juveniles ( } n=145) \text {, PFOA in negative } \\
\text { association with TSH }\end{array}$ & Lewis et al. $(2015)^{27)}$ \\
\hline
\end{tabular}

PFAS, perfluoroalkyl substance; PFOA, perfluorooctanoic acid; PFOS, perfluorooctane sulfonic acid; PFDeA, perfluorodecanoic acid; PFHXS, perfluorohexane sulfonic acid; PFNA, perfluorononanoic acid; TSH, thyroid stimulating hormone; fT3, free triiodothyronine; fT4, free thyroxine. 
levels of PFASs are lower compared to experimental studies or occupational settings, their thyroid disrupting effects could be compensated by the effects of other chemicals, if present.

\section{PFASs exposure and thyroid hormone disruption in susceptible human populations}

Many cross-sectional and often cohort studies have been conducted on susceptible populations including pregnant women and infants (Table 3 ). These studies reflect the importance of thyroid hormones that play crucial roles in developing fetus and infants. Unlike big observation studies employing nationally representative populations, these studies on susceptible groups are smaller in population size, but often measured more diverse types of PFASs.

Generally, exposure levels of PFASs showed negative associations with thyroid hormones but positive association with TSH, although deviations were often noted. Chemicals, Health and Pregnancy study conducted among pregnant women of Vancouver Canada ( $\mathrm{n}=152)$ reported a positive association between PFNA and TSH. Among TPOAb abnormal group, PFASs showed positive associations with TSH, and negative associations with $\mathrm{fT}^{355}$. In a mother and child cohort of Norway, PFASs such as perfluorodecanoic acid (PFDA) and perfluoroundecanoic acid (PFUnDA) showed also negative associations with T3, and PFOS showed a positive association with $\mathrm{TSH}^{36)}$. Similarly, in Norwegian pregnant women $(\mathrm{n}=930)$, PFOS that was measured at 18 weeks of pregnancy showed a positive association with $\mathrm{TSH}^{37)}$.

Hypothyroidism by PFASs exposure has also been reported in Asian countries. Among Taiwanese mothers of late pregnancy $(\mathrm{n}=285)$, maternal PFHxS levels showed a positive association with TSH, and PFASs such as PFNA, PFUnDA, or PFDoDA, showed negative associations with free and total $\mathrm{T} 4^{38}$. In addition, maternal PFASs showed negative associations with cord total T3 and T4 levels, and maternal PFDA showed a negative association with cord total T3 levels. In Chinese mother and child pairs recruited in Beijing $(\mathrm{n}=157)$, maternal perfluorododecanoic acid also showed negative associations with free and total thyroid hormones. In addition, the levels of long chain PFASs, e.g., PFOA, PFNA, PFDA, PFUnDA, PFDoDA, PFHxS, or PFOS in cord serum, showed negative associations with maternal tT3 levels ${ }^{39)}$.

However, in the same population of China, many long chain PFASs measured in maternal serum showed negative associations with maternal TSH, not supporting hypothyroidism effects of PFASs exposure ${ }^{39)}$. Some studies also reported similar inconsistent observations ${ }^{22,40,41)}$. In Hokkaido cohort with matched mother and child pairs ( $\mathrm{n}=39)$ ), PFOS levels measured in 24 weeks of pregnancy showed a negative association with maternal TSH, but a positive association with cord serum TSH. In another mother and child pair study ( $\mathrm{n}=83$ ), PFOA showed a positive association with $\mathrm{T} 4$ but only among female babies in the Netherlands $(\mathrm{n}=31)^{40)}$. The association between short chain PFASs exposure and thyroid hormone levels was also reported.
In a retrospective cohort study between 2006 and 2010 in Korea $(n=279)$, a positive association between PFPeA and thyroid hormones was observed, while PFHxS was also found to be positively associated in the same population ${ }^{41}$.

Unlike studies on nationally representative population studies, studies on pregnant women and matching infants often measured shorter or longer carbon chain PFASs and reported their associations with thyroid hormones ${ }^{39,41,42)}$. Especially for short carbon chain PFASs, however, limitations of representation of exposure windows should be considered in association studies because of their relatively shorter biological half-lives. Interpretation of cord thyroid hormone levels warrants caution as well, because cord hormone measurement may be influenced by thyroid hormones of maternal origin. Cord thyroid hormone levels can also be changing until shortly after the birth, by several delivery-related factors such as mode and time of delivery.

\section{Proposed mechanisms of thyroid disruption}

Most experimental studies and to lesser extent human observational studies support hypothyroidism effects of PFASs exposure, e.g., decrease of thyroid hormones, or increase of $\mathrm{TSH}^{5}$. PFASs can inhibit synthesis of thyroid hormones. PFASs are reported to decrease TPO activity in cells ${ }^{44)}$. These compounds can also modulate thyroid hormone signaling and function of nuclear hormone receptor. In addition, PFASs can influence metabolism and excretion of thyroid hormones. PFASs can compete with T4 for binding with transthyretin (TTR) which is a transporter of thyroid hormones, in rat. Binding of PFASs with TTR results in increase of fT4 levels in circulation, leading to facilitated metabolic excretion of this hormone ${ }^{45,46)}$. PFOS upregulates T4 metabolic genes in rat, and also enhance hepatic glucuronidation and subsequently excretion of $\mathrm{T}^{46)}$.

\section{Implications and conclusion}

Human observational studies show that PFASs exposure among general human population is associated with altered thyroid hormone levels. However, it should be noted that observational studies based on general human populations have several intrinsic limitations that should be considered in interpretation of the results. First, one cannot rule out potential effects of other TDCs. Phthalates, bisphenols, PCBs, OCPs, and PBDEs have been suggested as TDCs among general human populations ${ }^{47-49)}$. Exposure levels of PFASs among general populations tend to be low, and therefore, in the presence of other important TDCs that are not measured, the dose-response association can be significantly distorted often leading to wrong representation. Second, temporal comparability should be considered especially in cross-sectional or case-control studies ${ }^{5)}$. Since most long-chain PFASs have long biological half-lives, one time measurement of the exposure may reflect relatively long 
Table 3. Associations between exposure to PFASs and thyroid hormones among pregnant women or infants

\begin{tabular}{|c|c|c|c|c|}
\hline PFASS & Study population & Study period Thyroid measure & Association & Reference \\
\hline $\begin{array}{l}\text { PFHxS, PFOA, } \\
\text { PFOS, PFNA, } \\
\text { PFDeA, PFUnDA, } \\
\text { PFDoDA, PFHpA, } \\
\text { PFHxA }\end{array}$ & $\begin{array}{l}\text { Taiwan Maternal and } \\
\text { Infant Cohort Study } \\
\text { ( } 285 \text { mothers at 3rd } \\
\text { trimester, and } 116 \text { cord } \\
\text { blood) }\end{array}$ & 2000-2001 fT4, tT4, tT3, TSH & $\begin{array}{l}\text {-Among mothers, PFHxS in positive association } \\
\text { with TSH; maternal PFNA, PFUnDA, or PFDoDA } \\
\text { in negative associations with maternal fT4 and } \\
\text { tT4 } \\
\text {-Maternal PFNA, PFUnDA, or PFDoDA in } \\
\text { negative associations with cord tT4 and tT3; } \\
\text { Maternal PFDeA in negative association with }\end{array}$ & Wang et al. (2014) \\
\hline
\end{tabular}

\begin{tabular}{|c|c|c|c|c|c|}
\hline \multirow[b]{2}{*}{ PFOS, PFOA } & \multirow[b]{2}{*}{$\begin{array}{l}\text { Hokkaido Study on } \\
\text { the Environment and } \\
\text { Children's Health ( } 392 \\
\text { pairs) }\end{array}$} & \multirow{2}{*}{$2002-2005$} & & \multirow{2}{*}{ Kato et al. $(2016)^{22)}$} \\
\hline & & & fT4, TSH & $\begin{array}{l}\text {-PFOS in negative association with maternal } \\
\text { TSH, but positive association with cord TSH } \\
\text {-PFOA shows no association (mother during } 24 \\
\text { weeks of gestation and } 5 \text { days after delivery) }\end{array}$ & \\
\hline $\begin{array}{l}\text { PFOS, PFOA, } \\
\text { PFHXS, PFNA, } \\
\text { PFUnDA, PFHpS, } \\
\text { PFDeA }\end{array}$ & $\begin{array}{l}\text { Northern Norway } \\
\text { Mother and Child } \\
\text { Cohort Study. Pregnant } \\
\text { women (930) }\end{array}$ & 2003-2004 & $\mathrm{TSH}$ & $\begin{array}{l}\text {-PFOS in positive association with TSH } \\
\text { (18 weeks of gestation) }\end{array}$ & Wang et al. $(2013)^{37)}$ \\
\hline PFOS, PFOXS, PFOA & $\begin{array}{l}\text { Pregnant women, 2nd } \\
\text { trimester ( } 96 \text { cases, } \\
175 \text { controls) } \\
\text { Edmonton, Alberta, } \\
\text { Canada }\end{array}$ & $2005-2006$ & Hypo-thyroidism & -No association & Chan et al. $(2011)^{43)}$ \\
\hline $\begin{array}{l}\text { PFOA, PFHXS, } \\
\text { PFNA, PFOS }\end{array}$ & $\begin{array}{l}\text { Euthyroid pregnant } \\
\text { women (152) } \\
\text { Vancouver, Canada }\end{array}$ & $2007-2008$ & $\mathrm{fT} 4, \mathrm{tT} 4, \mathrm{TSH}$ & $\begin{array}{l}\text {-PFNA in positive association with TSH } \\
\text {-Among TPOAb normal group, no association } \\
\text {-Among TPOAb abnormal group ( } n=14) \text {, PFNA, } \\
\text { PFOS, or PFOA in positive associations with } \\
\text { TSH; all PFSAs in negative associations with fT4 }\end{array}$ & $\begin{array}{l}\text { Webster et al. } \\
\qquad(2014)^{35)}\end{array}$ \\
\hline 26 PFSAs & $\begin{array}{l}\text { Northern Norway } \\
\text { Mother and Child } \\
\text { Contaminant Cohort } \\
\text { Study. 2nd trimester } \\
\text { (441) }\end{array}$ & 2007-2009 & $\begin{array}{l}\text { fT3, fT4, tT3, tT4, } \\
\text { TSH, TBG, TTR, } \\
\text { Albumin, TBI, } \\
\text { TPOAb }\end{array}$ & $\begin{array}{l}\text {-PFOS in positive association with TSH } \\
\text {-PFDA or PFUnDA in negative association with } \\
\text { T3 }\end{array}$ & Berg et al. $(2015)^{36)}$ \\
\hline $\begin{array}{l}\text { PFHxS, PFHpS, } \\
\text { PFOS, PFOA, } \\
\text { PFNA, PFDeA, } \\
\text { PFUnDA, PFTrDA }\end{array}$ & $\begin{array}{l}\text { Pregnant women }(n=44) \\
\text { and infant }(n=43) \text { pair }\end{array}$ & $2008-2009$ & tT3, tT4, TSH & $\begin{array}{l}\text {-Maternal PFOS in negative association with } \\
\text { cord tT3 } \\
\text {-Maternal PFOA in positive association with } \\
\text { cord TSH } \\
\text {-Maternal PFTrDA in negative associations with } \\
\text { cord tT4 and tT3 }\end{array}$ & Kim et al. $(2011)^{42)}$ \\
\hline $\begin{array}{l}\text { PFOA, PFOS, } \\
\text { PFTrDA, PFHxS, } \\
\text { PFUnDA, PFNA, } \\
\text { PFPeA, PFDeA, } \\
\text { PFDoDA, PFTeDA }\end{array}$ & $\begin{array}{l}\text { Korea Ewha Birth \& } \\
\text { Growth Retrospective } \\
\text { Cohort (279) }\end{array}$ & 2006-2010 & $\mathrm{T} 3, \mathrm{~T} 4, \mathrm{TSH}$ & $\begin{array}{l}\text {-Among female infants, PFPeA, or PFHxS in } \\
\text { positive association with T3 or T4; PFNA in } \\
\text { negative association with TSH } \\
\text {-Among all infants, PFPeA in positive association } \\
\text { with T4 (PFSAs measured in maternal serum of } \\
\text { 24-28 weeks of gestations. Thyroid hormones } \\
\text { measured in cord blood serum) }\end{array}$ & $\begin{array}{l}\text { Shah-Kulkarni et al. } \\
\qquad(2016)^{41)}\end{array}$ \\
\hline $\begin{array}{l}\text { PFOA, PFOS, } \\
\text { other POPS }\end{array}$ & $\begin{array}{l}\text { Netherlands LINK study } \\
\text { (83 pairs) }\end{array}$ & $2011-2013$ & $\begin{array}{l}\text { T4 in heel prick } \\
\text { blood spots }\end{array}$ & $\begin{array}{l}\text {-Among girls ( } n=31 \text { ), PFOA in positive } \\
\text { association with T4 } \\
\text {-Among boys, no associations }\end{array}$ & de Cock et al. $(2014)^{40)}$ \\
\hline $\begin{array}{l}\text { PFHxS, PFOS, } \\
\text { PFOA, PFNA, } \\
\text { PFDeA, PFUnA, } \\
\text { PFDoA }\end{array}$ & $\begin{array}{l}\text { Beijing, China, } \\
\text { Mother-infant pair (157) }\end{array}$ & 2013 & $\begin{array}{l}\mathrm{fT} 3, \mathrm{fT} 4, \mathrm{tT} 3 \\
\mathrm{tT} 4, \mathrm{TSH}\end{array}$ & $\begin{array}{l}\text {-Maternal PFNA, PFDeA, PFUnDA, PFDoDA, or } \\
\text { PFOS in negative associations with maternal } \\
\text { TSH; Maternal PFDoA in negative associations } \\
\text { with fT3, tT3, fT4, or tT4 } \\
\text {-Cord PFOA, PFNA, PFDeA, PFUnDA, PFDoDA, } \\
\text { PFHxS, or PFOS in negative associations with } \\
\text { maternal tT3; cord PFOS in negative } \\
\text { association with maternal TSH }\end{array}$ & Yang et al. (2016) \\
\hline
\end{tabular}

PFAS, perfluoroalkyl substance; PFHxS, perfluorohexane sulfonic acid; PFOA, perfluorooctanoic acid; PFOS, perfluorooctane sulfonic acid; PFNA, perfluorononanoic acid; PFDeA, perfluorodecanoic acid; PFUnDA, perfluoroundecanoic acid; PFDoDA, perfluorododecanoic acid; PFHpA, perfluoroheptanoic acid; PFHxA, perfluorohexanoic acid; PFTrDA, perfluorotridecanoic acid; PFPeA, perfluoropentanoic acid; PFHpS, perfluoroheptane sulfonate; PFTeDA, perfluorotetradecanoic acid; PFUnA, perfluoroundecanoic acid; PFDoA, perfluorododecanoic acid; TSH, thyroid stimulating hormone; fT3, free triiodothyronine; fT4, free thyroxine; TPOAb, thyroid peroxidase antibody . 
period of exposure window. However, certain PFASs especially with shorter carbon chains have notably shorter biological half-lives and therefore lack temporal representativeness compared to their longer chain analogues. Moreover, influences of biological factors such as age and sex on effects of PFASs on thyroid hormones should add more complexity in elucidating underlying association.

Various PFASs were found to be associated with thyroid hormone levels among susceptible populations such as pregnant women and infants as well as general population. PFASs may affect synthesis, metabolism and excretion of thyroid hormones. Even within normal range, changes in thyroid hormone levels during sensitive periods of developments may cause permanent damages in intelligence and neurodevelopment. Because of their notable persistence and toxicity, some PFASs, such as PFOS and PFOA have been regulated worldwide. However, there are very limited information on their substituting analogues, e.g., shorter or longer carbon chain PFASs. For these substitutes, toxicity information is lacking and exposure levels have not been characterized. Currently available information based on association studies with such substituting PFASs suggests that these chemicals may also disrupt thyroid among pregnant women and infants. Further investigations including exposure assessment and toxicity studies, along with their associations with thyroid related health effects among susceptible human populations are warranted.

\section{Conflict of interest}

No potential conflict of interest relevant to this article was reported.

\section{Acknowledgments}

This study was supported by the Korea Ministry of Environment (MOE) as "the Environmental Health Action Program (RE201603033)".

\section{References}

1. Christensen DL, Baio J, Van Naarden Braun K, Bilder D, Charles J, Constantino JN, et al. Prevalence and characteristics of autism spectrum disorder among children aged 8 years: autism and developmental disabilities monitoring network, 11 sites, United States, 2012. MMWR Surveill Summ 2016;65:1-23.

2. Weintraub K. The prevalence puzzle: Autism counts. Nature 2011;479:22-4.

3. Bergman A, Heindel JJ, Jobling S, Kidd KA, Zoeller RT. State of the science of endocrine disrupting chemicals - 2012. Geneva: United Nations Environment Programme (UNEP) and World Health Organization (WHO), 2013.

4. Porterfield SP, Hendrich CE. The role of thyroid hormones in prenatal and neonatal neurological development: current perspectives. Endocr Rev 1993;14:94-106

5. Ballesteros V, Costa O, Iñiguez C, Fletcher T, Ballester F, Lopez-Espinosa MJ. Exposure to perfluoroalkyl substances and thyroid function in pregnant women and children: a systematic review of epidemiologic studies. Environ Int 2017;99:15-28.

6. Lyall K, Anderson M, Kharrazi M, Windham GC. Neonatal thyroid hormone levels in association with autism spectrum disorder. Autism Res 2016 Oct 14 [Epub]. https://doi. org/10.1002/aur.1708.

7. Korevaar TI, Muetzel R, Medici M, Chaker L, Jaddoe VW, de Rijke YB, et al. Association of maternal thyroid function during early pregnancy with offspring IQ and brain morphology in childhood: a population-based prospective cohort study. Lancet Diabetes Endocrinol 2016;4:35-43.

8. Boas M, Feldt-Rasmussen U, Main KM. Thyroid effects of endocrine disrupting chemicals. Mol Cell Endocrinol 2012;355:240-8.

9. Coperchini F, Awwad O, Rotondi M, Santini F, Imbriani M, Chiovato L. Thyroid disruption by perfluorooctane sulfonate (PFOS) and perfluorooctanoate (PFOA). J Endocrinol Invest 2017;40:105-21.

10. Naile JE, Wiseman S, Bachtold K, Jones PD, Giesy JP. Transcriptional effects of perfluorinated compounds in rat hepatoma cells. Chemosphere 2012;86:270-7.

11. Buck RC, Franklin J, Berger U, Conder JM, Cousins IT, de Voogt P, et al. Perfluoroalkyl and polyfluoroalkyl substances in the environment: terminology, classification, and origins. Integr Environ Assess Manag 2011;7:513-41.

12. Kissa E. Fluorinated surfactants and repellants. 2nd ed. New York: Marcel Dekker Inc., 2001.

13. Chang SC, Das K, Ehresman DJ, Ellefson ME, Gorman GS, Hart JA, et al. Comparative pharmacokinetics of perfluorobutyrate in rats, mice, monkeys, and humans and relevance to human exposure via drinking water. Toxicol Sci 2008; 104:40-53.

14. Nilsson H, Kärrman A, Westberg H, Rotander A, van Bavel B, Lindström G. A time trend study of significantly elevated perfluorocarboxylate levels in humans after using fluorinated ski wax. Environ Sci Technol 2010;44:2150-5.

15. Olsen GW, Burris JM, Ehresman DJ, Froehlich JW, Seacat AM, Butenhoff JL, et al. Half-life of serum elimination of perfluorooctanesulfonate,perfluorohexanesulfonate, and perfluorooctanoate in retired fluorochemical production workers. Environ Health Perspect 2007;1 15:1298-305.

16. Olsen GW, Chang SC, Noker PE, Gorman GS, Ehresman DJ, Lieder PH, et al. A comparison of the pharmacokinetics of perfluorobutanesulfonate (PFBS) in rats, monkeys, and humans. Toxicology 2009;256:65-74.

17. Chengelis CP, Kirkpatrick JB, Myers NR, Shinohara M, Stetson PL, Sved DW. Comparison of the toxicokinetic behavior of perfluorohexanoic acid (PFHxA) and nonafluorobutane-1-sulfonic acid (PFBS) in cynomolgus monkeys and rats. Reprod Toxicol 2009;27:400-6.

18. Ohmori K, Kudo N, Katayama K, Kawashima Y. Comparison of the toxicokinetics between perfluorocarboxylic 
acids with different carbon chain length. Toxicology 2003; 184:135-40.

19. Tatum-Gibbs K, Wambaugh JF, Das KP, Zehr RD, Strynar $\mathrm{MJ}$, Lindstrom $\mathrm{AB}$, et al. Comparative pharmacokinetics of perfluorononanoic acid in rat and mouse. Toxicology 2011;281:48-55.

20. Sundström M, Chang SC, Noker PE, Gorman GS, Hart JA, Ehresman DJ, et al. Comparative pharmacokinetics of perfluorohexanesulfonate (PFHxS) in rats, mice, and monkeys. Reprod Toxicol 2012;33:441-51.

21. Chang SC, Noker PE, Gorman GS, Gibson SI, Hart JA, Ehresman DJ, et al. Comparative pharmacokinetics of perfluorooctanesulfonate (PFOS) in rats, mice, and monkeys. Reprod Toxicol 2012;33:428-40.

22. Kato S, Itoh S, Yuasa M, Baba T, Miyashita C, Sasaki S, et al. Association of perfluorinated chemical exposure in utero with maternal and infant thyroid hormone levels in the Sapporo cohort of Hokkaido Study on the Environment and Children's Health. Environ Health Prev Med 2016;21: 334-44.

23. Kang H, Choi K, Lee HS, Kim DH, Park NY, Kim S, et al. Elevated levels of short carbon-chain PFCAs in breast milk among Korean women: Current status and potential challenges. Environ Res 2016;148:351-9.

24. Eriksson U, Mueller JF, Toms LL, Hobson P, Kärrman A. Temporal trends of PFSAs, PFCAs and selected precursors in Australian serum from 2002 to 2013. Environ Pollut 2017;220(Pt A):168-77.

25. Melzer D, Rice N, Depledge MH, Henley WE, Galloway TS. Association between serum perfluorooctanoic acid (PFOA) and thyroid disease in the U.S. National Health and Nutrition Examination Survey. Environ Health Perspect 2010;118:686-92.

26. Wen LL, Lin LY, Su TC, Chen PC, Lin CY. Association between serum perfluorinated chemicals and thyroid function in U.S. adults: the National Health and Nutrition Examination Survey 2007-2010. J Clin Endocrinol Metab 2013;98:E1456-64.

27. Lewis RC, Johns LE, Meeker JD. Serum biomarkers of exposure to perfluoroalkyl substances in relation to serum testosterone and measures of thyroid function among adults and adolescents from NHANES 2011-2012. Int J Environ Res Public Health 2015;12:6098-114.

28. Webster GM, Rauch SA, Marie NS, Mattman A, Lanphear BP, Venners SA. Cross-sectional associations of serum perfluoroalkyl acids and thyroid hormones in U.S. adults: variation according to TPOAb and iodine status (NHANES 2007-2008). Environ Health Perspect 2016;124:935-42.

29. Jain RB. Association between thyroid profile and perfluoroalkyl acids: data from NHNAES 2007-2008. Environ Res 2013;126:51-9.

30. Ji K, Kim S, Kho Y, Paek D, Sakong J, Ha J, et al. Serum concentrations of major perfluorinated compounds among the general population in Korea: dietary sources and potential impact on thyroid hormones. Environ Int 2012;45:78-85.
31. Harada K, Inoue K, Morikawa A, Yoshinaga T, Saito N, Koizumi A. Renal clearance of perfluorooctane sulfonate and perfluorooctanoate in humans and their speciesspecific excretion. Environ Res 2005;99:253-61.

32. Krassas GE, Poppe K, Glinoer D. Thyroid function and human reproductive health. Endocr Rev 2010;31:702-55.

33. Wiens SC, Eales JG. The effects of $17 \beta$-estradiol injections on thyroid hormone deiodination pathways in liver and other tissues of female and male rainbow trout (Oncorhynchus mykiss) at different stages of sexual maturity. Can J Zool 2005;83:596-603.

34. Leatherland JF. Effects of 17 beta-estradiol and methyl testosterone on the activity of the thyroid gland in rainbow trout, Salmo gairdneri Richardson. Gen Comp Endocrinol 1985;60:343-52.

35. Webster GM, Venners SA, Mattman A, Martin JW. Associations between perfluoroalkyl acids (PFASs) and maternal thyroid hormones in early pregnancy: a population-based cohort study. Environ Res 2014;133:338-47.

36. Berg V, Nøst TH, Hansen S, Elverland A, Veyhe AS, Jorde $\mathrm{R}$, et al. Assessing the relationship between perfluoroalkyl substances, thyroid hormones and binding proteins in pregnant women; a longitudinal mixed effects approach. Environ Int 2015;77:63-9.

37. Wang Y, Starling AP, Haug LS, Eggesbo M, Becher G, Thomsen C, et al. Association between perfluoroalkyl substances and thyroid stimulating hormone among pregnant women: a cross-sectional study. Environ Health 2013;12:76.

38. Wang Y, Rogan WJ, Chen PC, Lien GW, Chen HY, Tseng YC, et al. Association between maternal serum perfluoroalkyl substances during pregnancy and maternal and cord thyroid hormones: Taiwan maternal and infant cohort study. Environ Health Perspect 2014;122:529-34.

39. Yang L, Li J, Lai J, Luan H, Cai Z, Wang Y, et al. Placental transfer of perfluoroalkyl substances and associations with thyroid hormones: Beijing Prenatal Exposure Study. Sci Rep 2016;6:21699.

40. de Cock M, de Boer MR, Lamoree M, Legler J, van de Bor M. Prenatal exposure to endocrine disrupting chemicals in relation to thyroid hormone levels in infants: a Dutch prospective cohort study. Environ Health 2014;13:106.

41. Shah-Kulkarni S, Kim BM, Hong YC, Kim HS, Kwon EJ, Park H, et al. Prenatal exposure to perfluorinated compounds affects thyroid hormone levels in newborn girls. Environ Int 2016;94:607-13.

42. Kim S, Choi K, Ji K, Seo J, Kho Y, Park J, et al. Transplacental transfer of thirteen perfluorinated compounds and relations with fetal thyroid hormones. Environ Sci Technol 2011;45:7465-72..

43. Chan E, Burstyn I, Cherry N, Bamforth F, Martin JW. Perfluorinated acids and hypothyroxinemia in pregnant women. Environ Res 2011;111:559-64.

44. Song M, Kim YJ, Park YK, Ryu JC. Changes in thyroid peroxidase activity in response to various chemicals. J Environ Monit 2012;14:2121-6. 
45. Weiss JM, Andersson PL, Lamoree MH, Leonards PE, van Leeuwen SP, Hamers T. Competitive binding of polyand perfluorinated compounds to the thyroid hormone transport protein transthyretin. Toxicol Sci 2009;109:20616.

46. Yu WG, Liu W, Jin YH. Effects of perfluorooctane sulfonate on rat thyroid hormone biosynthesis and metabolism. Environ Toxicol Chem 2009;28:990-6.

47. Park C, Choi W, Hwang M, Lee Y, Kim S, Yu S, et al. Associations between urinary phthalate metabolites and bisphenol A levels, and serum thyroid hormones among the Korean adult population - Korean National Environmental Health
Survey (KoNEHS) 2012-2014. Sci Total Environ 2017;5845:950-7.

48. Kim S, Park J, Kim HJ, Lee JJ, Choi G, Choi S, et al. Association between several persistent organic pollutants and thyroid hormone levels in cord blood serum and bloodspot of the newborn infants of Korea. PLoS One 2015; 10:e0125213.

49. Kim S, Park J, Kim HJ, Lee JJ, Choi G, Choi S, et al. Association between several persistent organic pollutants and thyroid hormone levels in serum among the pregnant women of Korea. Environ Int 2013;59:442-8. 